\title{
Prevalence, antimicrobial susceptibility and genotypic characteristics of Staphylococcus aureus in Tanzania: a systematic review
}

Tutu Mzee ( $\nabla$ tmzee@ihi.or.tz )

Ifakara Health Institute https://orcid.org/0000-0002-5918-0574

Theckla Kazimoto

Ifakara Health Institute

Joseph Madata

Ifakara Health Institute

Rose Masalu

University of Dar es Salaam

Markus Bischoff

Universitat des Saarlandes

Mecky Matee

Muhimbili University of Health and Allied Sciences

Sören Becker

Universitat des Saarlandes

\section{Research article}

Keywords: Staphylococcus aureus; Prevalence; Antimicrobial resistance; Genotyping; Infection; Colonization; Tanzania

Posted Date: March 12th, 2020

DOI: https://doi.org/10.21203/rs.3.rs-16889/v1

License: (9) This work is licensed under a Creative Commons Attribution 4.0 International License.

Read Full License

Version of Record: A version of this preprint was published at Bulletin of the National Research Centre on September 27th, 2021. See the published version at https://doi.org/10.1186/s42269-021-00612-z. 


\section{Abstract}

Background : Information about prevalence, antibiotic resistance and genotyping data of Staphylococcus aureus (S aureus) isolated from different infections and colonization, both in humans and animals is scarce in Tanzania. Given the wide range of infections $S$ aureus can cause and the raise of methicillin resistant $S$ aureus (MRSA) globally, this review was aimed at collecting local published data on the $S$ aureus bacterium to better understand the epidemiology. Methodology: A systematic review of scientific studies reporting on prevalence, antibiotic resistance and genotyping of $S$ aureus in human and animal infection and colonization was done. Literatures extracted from electronic databases such as PubMed and Google Scholar were screened for eligibility and relevant articles included into the review. The review is limited to literatures published in English, between the years 2010 and 2018. . Results: 39 studies conducted in 5 of the 9 administrative zones in Tanzania were reviewed to establish S aureus prevalence in human and animal infection and colonization. Prevalence in human ranged from $1-60 \%$. Antibiotic resistance patterns of $S$ aureus isolated from colonized humans showed higher resistance rates against erythromycin (49\%), co-trimoxazole (38\%) and tetracycline (28\%) compared to report from other studies outside Africa. The review suggests an increased MRSA prevalence of up to $33 \%$ compared to $16 \%$ reported in previous years. Genotypic data reviewed suggested that MRSA predominantly belonged to ST88. S aureus prevalence in animal studies ranged from $33-49 \%$ whereas MRSA prevalence ranged from $4-35 \%$. Generally low antibiotic resistance levels were reported in these studies with exception to penicillin (85\%) and ampicillin (73\%). Resistance against tetracycline reported at $30 \%$ collaborates the suggested overuse of the drug in livestock keeping in Tanzania. Conclusion : Prevalence of MRSA in Tanzania is rising although clear variations between different geographic areas could be observed. Nonsusceptibility to commonly prescribed antibiotics in community associated $\mathrm{S}$ aureus is of concern. Research strategies to better understand the $S$ aureus epidemiology by including genotypic characterisations, as well as strengthening antimicrobial resistance surveillance and antimicrobial stewardship are recommended.

\section{Background}

Antimicrobial resistance (AMR) is a global concern estimated to account for approximately 700,000 deaths each year (1). If no appropriate measures are taken to slowdown the progression of this epidemic it is estimated that by 2050 it will cost the world around 10 million lives per annum (1). The lack of development of new antimicrobial agents in the pipeline further emphasises the necessity to reduce dependency on antibiotics by implementing infection control strategies $(1,2)$.

According to the 2014 World Health Organization (WHO) report, most regions stated over $50 \%$ bacterial resistance against third generation antibiotics, particularly methicillin resistant $S$. aureus (MRSA) clones in hospitals (2). Furthermore in 2017, the WHO listed antibiotic resistant bacteria including Staphylococcus aureus (S. aureus) as a priority bacterium to guide research, discovery and development of new antibiotics (3). The report further recommended special attention to be directed towards the 
identified resistant bacteria due to their ability to rapidly develop resistance against multiple antibiotic classes hence limiting therapeutic options (3).

S. aureus is an old bacterium discovered in the $18^{\text {th }}$ Century, antibiotic resistance in the bacterium against penicillin was described in the 1950's, the penicillin-resistant staphylococci inactivates penicillin function by an enzyme called penicillinase or beta-lactamase which degrades the $\beta$-lactam ring in penicillin, thus altering the shape of penicillin and preventing its binding to the penicillin binding proteins (4). Following penicillin failure in treating $S$ aureus infections, methicillin was introduced. Methicillin was particularly effective upon its introduction into clinical therapy due to its ability to resists the action of $\beta$-lactamase. However, methicillin resistance in $S$. aureus was also reported only a year after its introduction. Strains of $S$. aureus resistant to methicillin or oxacillin or other $\beta$-lactam compounds are still termed MRSA, only to honour the historic role of methicillin that used to effectively treat staphylococcal infections, and it is to methicillin that the resistance was first described (5). Several terms are used to describe different MRSA strains associated with outbreaks in different settings based on strains' genomic background and level of virulence (6). MRSA strains associated with hospital-acquired infections are commonly abbreviated to HA-MRSA, while strains causing community-associated infection are abbreviated to CA-MRSA and those associated with livestock are abbreviated as LA-MRSA.

S. aureus, is both a commensal and potentially harmful pathogen in humans and animals. The bacterium can give rise to a variety of infections ranging from mild skin and soft tissue infections to more serious and complex diseases such as pneumonia, septicaemia, infective endocarditis and other deep-seated infections (e.g., osteomyelitis) in humans as well as mastitis and necrotic infections in a variety of animal hosts. S. aureus can also colonise the skin and approximately $30 \%$ of the population is found to be transiently colonised if nasal swabs are examined microbiologically.

High levels of MRSA have been reported across Africa, ranging between $43 \%$ and $72 \%$, as reported in Cameroon, South Africa and Ethiopia (7). The vast majority of clinical $S$ aureus/MRSA data in Africa is associated with hospital acquired infection ( $\mathrm{HAl}$ ) affecting individuals with healthcare related risk such as recent hospitalization, surgery and underlying chronic diseases (8). Information on community acquired $S$ aureus/MRSA infections (CAI) causing diseases in people with no healthcare associated risk is also available on the continent even though to a lesser extent. Moreover very limited data on livestock acquired $S$ aureus/MRSA infections (LAl) whereby $S$. aureus clones of animal origins colonize or cause infections in humans have been published in Africa (7). Further information on the antibiotic resistant clones occurring in both human and animal are very limited, hindering comprehensive understanding of the epidemiology of the bacterium (9).

Tanzania, a developing country located along the East African coast, housing a population of about 57 million people, of which $36 \%$ is engaged in livestock keeping as the major source of livelihood. The potential for human-animal contact is very high, especially in tribes that co-habit with their animals such as Maasai people. The aim of this review is to summarize literatures reporting on the prevalence, antibiotic resistance of $S$. aureus in Tanzania. Furthermore reported genotypic characterization of $S$. 
aureus will be reviewed to provide a more nuanced profile of $S$. aureus genetic diversity in colonization and infections both in animal and human hosts as well as their possible relationship.

\section{Materials And Methods}

\section{Eligibility criteria}

A systematic review of Tanzanian scientific studies reporting on prevalence, antimicrobial resistance and genotypic characterization of $S$. aureus from human and animal sources published between 2010 and 2018 was performed. Information sought for in the publication included commensal and clinical $S$. aureus recovered from different infections including invasive and non-invasive. Animal studies reporting on $S$. aureus recovered from animals and their products were also considered. All studies that recovered less than five $S$. aureus isolates in their studies as well as publications that were written in a language other than English were excluded from this review.

\section{Information sources and search strategies}

PubMed database was researched in November 2018 by a librarian using Boolean operators "AND" and "OR" to identify studies fitting our inclusion criteria. The following search terms were used

"Staphylococcus aureus OR S. aureus AND antimicrobial susceptibility OR antibiotic resistance AND prevalence AND Tanzania." Additionally search words such as "Molecular typing AND S. aureus AND antibiotic resistance AND Tanzania" and "Staphylococcus aureus OR S aureus AND antimicrobial susceptibility OR antibiotic resistance AND prevalence AND Tanzania" were used in Google scholar to identify eligible articles. The bibliographies of all eligible documents were hand-searched for additional publications eligible for review.

\section{Data extraction and appraisal process}

The Joana Briggs Institute checklist was applied to appraise and review the quality of each study accessed by two independent reviewers. A data extraction form was designed to capture required information such as author, year of publication, study period, methodology, MRSA identification strategies, $S$. aureus and MRSA prevalence, antibiotic resistance patterns as well as genotypic information. Two reviewers were involved in the process whereby the first reviewer (TM) extracted the information and the second reviewer (TK) double checked the information to eliminate possible bias. All disagreement raised during the critical appraisal process were resolved through reviewers discussions. All extracted manuscripts were analyzed using reference manager ENDNOTEX7. This study followed the standardized scientific writing format of the Preferred Reporting Items for Systematic Reviews and MetaAnalyses (PRISMA) guidelines. The study has not taken on any meta-analysis due to the heterogeneity of the studies under review; nevertheless mean resistance rates and prevalence were calculated to help present the results better.

\section{Scope of the study}


This review is limited to the prevalence, antibiotic resistance patterns and genetic typing information (i.e. resistance genes, virulence genes, Staphylococcus protein A (spa) typing and multilocus sequence typing) of $S$. aureus retrieved from human and animal hosts.

\section{Results And Discussion.}

PubMed search resulted in 16 eligible articles followed by an additional 8 articles included from the Googlescholar search. Rigorous reference list review supplemented another 14 eligible publications making a total of 39 (Figure 1).

Data concerning $S$. aureus prevalence, antimicrobial resistance and genotyping were extracted from studies performed in 5 zones of the 9 administrative zones in Tanzania. Table 1, describes the distribution of the published articles review in their consecutive zones. The majority of the publications reviewed were from regions in the eastern and lake zones (i.e. $43.6 \%$ and $30.8 \%$ respectively), showing lack of $S$. aureus researched data in the other parts of the country figure

Table 1: Distribution of the reviewed articles by zone in Tanzania.

\begin{tabular}{|l|c|c|c|}
\hline Zone & Human & Animal & Human \& Animal \\
\hline Eastern Zone & 14 & 2 & 1 \\
\hline Lake Zone & 12 & - & - \\
\hline Northern Zone & 5 & 1 & - \\
\hline Central & 1 & - & - \\
\hline Zanzibar & 2 & 1 & - \\
\hline Total & 34 & 4 & 1 \\
\hline
\end{tabular}

Majority of the publications were reported in the eastern and lake zones. S. aureus data of animal origin have been poorly represented throughout the country

\section{Prevalence, antibiotic resistance and genotyping of $S$. aureus isolated from humans}

S. aureus prevalence was reported in thirty three (33) human related studies, gave an account which ranged from $11-60 \%$ as summarized in Table

2. Higher infection rates were typically observed in SSTI's nevertheless the bacterium was also implicated in other infections. Furthermore it was notably observed that $S$. aureus colonization ranged between $27 \%-60 \%$ in the three zones that reported on colonization. Since most colonization studies involved healthy individuals these finding are indicative of the considerable circulation of community associated $S$. aureus in the country.

The publication under review showed that $S$. aureus was isolated in a number of infections including SSTI's, bloodstream, otitis media, respiratory tract and urinary tract infections (Figure 3 ) staying true to characteristically causing a wide range of infections. It was also evident how various vulnerable populations such as children, elderly and immune compromised individual were at risk of $S$. aureus infections/colonization. This observation emphasises the necessity to establish a reliable and sustainable surveillance system to monitor the $S$. aureus bacterium countrywide. 
Table 2: Human related studies investigating $S$. aureus in different conditions and populations across Tanzania 


\begin{tabular}{|c|c|c|c|c|c|c|}
\hline S.No & $\begin{array}{l}\text { Prevalence } \\
\text { S. aureus \% (n) }\end{array}$ & $\begin{array}{l}\text { Prevalence } \\
\text { MRSA \% } \\
\text { (n) }\end{array}$ & Population & $\begin{array}{l}\text { Source of } \\
S \\
\text { aureus isolates }\end{array}$ & Location & Reference \\
\hline 1 & $41.4 \%(157)$ & $37.6 \%(59)$ & $\begin{array}{l}\text { Health care workers } \\
\text { adults male and } \\
\text { female from } \\
\text { June } 2016 \text {-October } \\
2016 .\end{array}$ & Nasal carriage & $\begin{array}{l}\text { Dar es } \\
\text { Salaam } \\
\text { (Eastern } \\
\text { zone) }\end{array}$ & $\begin{array}{l}\text { Joachim A } \\
\text { et al } \\
2018(11)\end{array}$ \\
\hline 2 & NR & $\begin{array}{l}11.8 \% \text { in } \\
\text { ICU } \\
\text { patients and } \\
2.1 \% \text { in ICU } \\
\text { HCW. }\end{array}$ & $\begin{array}{l}\text { ICU patients (male } \\
\text { and female of all age } \\
\text { groups) and ICU } \\
\text { HCW from October } \\
2012 \text { - March } 2013\end{array}$ & Nasal carriage & $\begin{array}{l}\text { Dar es } \\
\text { Salaam } \\
\text { (Eastern } \\
\text { zone) }\end{array}$ & $\begin{array}{l}\text { Geofrey A } \\
\text { et al } \\
2015 \text { ( } 12)\end{array}$ \\
\hline 3 & $\begin{array}{l}34.5 \%(89) \text { in } \\
\text { swabs collected on } \\
\text { admission. } \\
20 \% \text { (4) in swabs } \\
\text { collected } 48 \mathrm{hrs} \\
\text { after admission }\end{array}$ & $\begin{array}{l}24.7 \%(22) \\
\text { from } \\
\text { samples on } \\
\text { admission, } \\
\text { and } 50 \%(2) \\
\text { in samples } \\
\text { collected } \\
\text { after } 48 \mathrm{hrs.}\end{array}$ & $\begin{array}{l}\text { Patients admitted to } \\
\text { emergency or } \\
\text { medical ward aged } 5 \\
\text { and above. }\end{array}$ & Nasal carriage & $\begin{array}{l}\text { Dar es } \\
\text { Salaam } \\
\text { (Eastern } \\
\text { zone) }\end{array}$ & $\begin{array}{l}\text { Joachim A } \\
\text { et al } \\
2017 \text { ( } 13)\end{array}$ \\
\hline 4 & $40 \%(114)$ & $8.3 \%(12)$ & $\begin{array}{l}\text { Healthy children } \\
<5 y r s \\
\text { From April } 2010 \text { - } \\
\text { June } 2010\end{array}$ & Nasal carriage & $\begin{array}{l}\text { Dar es } \\
\text { Salaam } \\
\text { (Eastern } \\
\text { zone) }\end{array}$ & $\begin{array}{l}\text { Moyo S et } \\
\text { al } 2014 \text { ( } \\
14)\end{array}$ \\
\hline 5 & $\begin{array}{l}\text { Overall } 23.2 \% \\
\text { ( } 223 \text { ), where by } \\
26.2 \% \\
\text { (95), detected in } \\
\text { children with acute } \\
\text { respiratory } \\
\text { infection and } \\
21.4 \% \text { (128) were } \\
\text { detected in } \\
\text { children without } \\
\text { acute respiratory } \\
\text { infection }\end{array}$ & NR & $\begin{array}{l}\text { ages } 2 \text { to } 10 \text {, with } \\
\text { axillary temperature } \\
\text { of } \leq 38^{0} \mathrm{C} \text { Dar es } \\
\text { Salaam from April to } \\
\text { August } 2008 \text { (DSM) } \\
\text { and June to } \\
\text { December } 2008 \\
\text { (Ifakara) }\end{array}$ & Nasal carriage & $\begin{array}{l}\text { Dar es } \\
\text { Salaam } \\
\text { and } \\
\text { Morogoro } \\
\text { (Eastern } \\
\text { zone) }\end{array}$ & $\begin{array}{l}\text { Chochua et } \\
\text { al } 2016 \text { ( } \\
15)\end{array}$ \\
\hline 6 & $13.2 \%(138)$ & NR & $\begin{array}{l}\text { Healthy children } \\
<5 y r s \text { born after } \\
\text { mass distribution of } \\
\text { Azithromycin for } \\
\text { tranchoma control. } \\
\text { Study was conducted } \\
\text { in } 2014\end{array}$ & Nasal carriage & $\begin{array}{l}\text { Kilosa, } \\
\text { Morogoro } \\
\text { (Eastern } \\
\text { zone) }\end{array}$ & $\begin{array}{l}\text { Bloch EM } \\
\text { et al } \\
2017(16)\end{array}$ \\
\hline 7 & $22 \%(22)$ & $0 \%$ & $\begin{array}{l}100 \text { nasal swabs } \\
\text { from healthy } \\
\text { individuals with no } \\
\text { epidemiological } \\
\text { connection were } \\
\text { collected within } \\
\text { urban and peri urban } \\
\text { Morogoro } \\
\text { Municipalities }\end{array}$ & Nasal carriage & $\begin{array}{l}\text { Morogoro } \\
\text { (Eastern } \\
\text { zone) }\end{array}$ & $\begin{array}{l}\text { Katakweba } \\
\text { et al } \\
2016 \text { ( 17) }\end{array}$ \\
\hline
\end{tabular}




\begin{tabular}{|c|c|c|c|c|c|c|}
\hline 8 & $13.2 \%(245)$ & $\begin{array}{l}23.3 \% \\
(57)\end{array}$ & $\begin{array}{l}\text { Male and female of } \\
\text { all age groups who } \\
\text { were subjected to } \\
\text { microbiology testing } \\
\text { between January } \\
2005 \text { and December } \\
2009 \text { (Retrospective } \\
\text { study). }\end{array}$ & Bacteremia & $\begin{array}{l}\text { Dar es } \\
\text { Salaam } \\
\text { (Eastern } \\
\text { zone) }\end{array}$ & $\begin{array}{l}\text { Moyo S et } \\
\text { al } 2010 \text { ( } \\
18)\end{array}$ \\
\hline 9 & $28 \%(12)$ & NR & $\begin{array}{l}\text { Male and female } \\
\text { sickle cell anemic } \\
\text { patients of all age } \\
\text { groups seeking } \\
\text { health care at MNH } \\
\text { from January 2006- } \\
\text { December } 2008 \\
\end{array}$ & Bacteremia & $\begin{array}{l}\text { Dar es } \\
\text { Salaam } \\
\text { (Eastern } \\
\text { zone) }\end{array}$ & $\begin{array}{l}\text { Makani J } \\
\text { et al } \\
2015 \text { ( 19) }\end{array}$ \\
\hline 10 & $\begin{array}{l}\text { In blood } 36.5 \% \\
(27) \text {, and in pus } \\
\text { swabs } 52.3 \% \\
(132)\end{array}$ & NR & $\begin{array}{l}\text { Neonates aged } 3 \text { - } 26 \\
\text { days suspected with } \\
\text { neonatal sepsis. } \\
\text { Study duration } \\
\text { October } 2009 \text { - } \\
\text { January } 2010\end{array}$ & $\begin{array}{l}\text { Bacteremia } \\
\text { and wound } \\
\text { swabs }\end{array}$ & $\begin{array}{l}\text { Dar es } \\
\text { Salaam } \\
\text { (Eastern } \\
\text { zone) }\end{array}$ & $\begin{array}{l}\text { Mhada T } \\
\text { et al } \\
2012 \text { ( 20) }\end{array}$ \\
\hline 11 & $12.2 \%(18)$ & $44.4 \%(8)$ & $\begin{array}{l}100 \text { participants } \\
\text { (male and female } \\
\text { aged 18-80) with } \\
\text { clinical evidence of } \\
\text { Surgical site } \\
\text { infection. Duration } \\
\text { from September } \\
2011 \text { to February } \\
2012\end{array}$ & $\begin{array}{l}\text { Wound } \\
\text { infections }\end{array}$ & $\begin{array}{l}\text { Dar es } \\
\text { Salaam } \\
\text { (Eastern } \\
\text { zone) }\end{array}$ & $\begin{array}{l}\text { Manyahi } \\
\text { Jet al } \\
2014 \text { ( 21) }\end{array}$ \\
\hline 12 & $71.4 \%(132)$ & $0.8 \%(1)$ & $\begin{array}{l}\text { Skin and soft tissue } \\
\text { infections patients of } \\
\text { all age groups }\end{array}$ & $\begin{array}{l}\text { Wound } \\
\text { infections }\end{array}$ & $\begin{array}{l}\text { Bagamoyo } \\
\text { (Eastern } \\
\text { zone) }\end{array}$ & $\begin{array}{l}\text { Kazimoto } \\
\text { T et al } \\
2018 \text { ( 22) }\end{array}$ \\
\hline 13 & $48.3 \%(131)$ & NR & $\begin{array}{l}\text { Asymptomatic otitis } \\
\text { media (OM)- } \\
\text { associated bacteria } \\
\text { found in patients } \\
\text { living with HIV }\end{array}$ & $\begin{array}{l}\text { Ear } \\
\text { colonization }\end{array}$ & $\begin{array}{l}\text { Morogoro } \\
\text { (Eastern } \\
\text { zone) }\end{array}$ & $\begin{array}{l}\text { Mwabete } \\
\text { KD et al } \\
2018 \text { ( 23) }\end{array}$ \\
\hline 14 & $\begin{array}{l}\text { The overall } \\
\text { carriage rate of S. } \\
\text { aureus was } 21.0 \% \\
\text { (66). } 47 \% \text { (31) } \\
\text { were from } \\
\text { preclinical } \\
\text { students while } 53 \\
\% \text { (35) from } \\
\text { clinical students }\end{array}$ & $1.5 \%(1)$ & $\begin{array}{l}\text { Healthy students } \\
\text { (clinical and Pre } \\
\text { clinical) aged } 18 \text { and } \\
\text { above. Study } \\
\text { duration February to } \\
\text { June } 2013\end{array}$ & Nasal carriage & $\begin{array}{l}\text { Mwanza } \\
\text { (Lake } \\
\text { zone) }\end{array}$ & $\begin{array}{l}\text { Okamo B } \\
\text { et al } \\
2016(24)\end{array}$ \\
\hline 15 & $21.5 \%$ & $28 \%(9)$ & $\begin{array}{l}300 \text { neonates with } \\
\text { clinical neonatal } \\
\text { sepsis }\end{array}$ & Bacteremia & $\begin{array}{l}\text { Mwanza } \\
\text { (Lake } \\
\text { zone) }\end{array}$ & $\begin{array}{l}\text { Kayange N } \\
\text { et al } \\
2010 \text { ( 25) }\end{array}$ \\
\hline 16 & $14.8(8)$ & $50 \%(4)$ & $\begin{array}{l}402 \text { malnourished } \\
\text { under five children. }\end{array}$ & Bacteremia & $\begin{array}{l}\text { Mwanza } \\
\text { Lake zone }\end{array}$ & $\begin{array}{l}\text { Ahmed M } \\
\text { et al } \\
2017 \text { ( 26) }\end{array}$ \\
\hline
\end{tabular}




\begin{tabular}{|c|c|c|c|c|c|c|}
\hline & & & $\begin{array}{l}\text { Study duration } \\
\text { September 2012- } \\
\text { January } 2013 .\end{array}$ & & & \\
\hline 17 & $13.7 \%(29)$ & 79\% (23) & $\begin{array}{l}\text { Patients of all age } \\
\text { groups and gender } \\
\text { with lower limb } \\
\text { ulcers seen at the } \\
\text { surgical ward or } \\
\text { outpatient } \\
\text { department from } \\
\text { November 2010-April } \\
2012\end{array}$ & $\begin{array}{l}\text { Wound } \\
\text { infection }\end{array}$ & $\begin{array}{l}\text { Mwanza } \\
\text { (Lake } \\
\text { zone) }\end{array}$ & $\begin{array}{l}\text { Mbunda F } \\
\text { et al } \\
2012 \text { ( 27) }\end{array}$ \\
\hline 18. & $8.9 \%(18)$ & $\begin{array}{l}44.4 \% \\
\text { (8) }\end{array}$ & $\begin{array}{l}\text { Patients of all age } \\
\text { groups with chronic } \\
\text { lower limb ulcers } \\
\text { seen at the surgical } \\
\text { ward.300 wound } \\
\text { infection were } \\
\text { swabbed between } \\
\text { November } 2011 \text { and } \\
\text { February } 2012\end{array}$ & $\begin{array}{l}\text { Wound } \\
\text { infection }\end{array}$ & $\begin{array}{l}\text { Mwanza } \\
\text { (Lake } \\
\text { zone) }\end{array}$ & $\begin{array}{l}\text { Moremi et } \\
\text { al } 2014 \text { ( } \\
28)\end{array}$ \\
\hline 19 & $28.6 \%(18)$ & $19 \%(3)$ & $\begin{array}{l}65 \text { patients of all age } \\
\text { groups who } \\
\text { underwent major } \\
\text { surgery at BMC } \\
\text { between July } 2009 \\
\text { and June } 2010\end{array}$ & $\begin{array}{l}\text { Surgical site } \\
\text { wound } \\
\text { infection }\end{array}$ & $\begin{array}{l}\text { Mwanza } \\
\text { (Lake } \\
\text { zone) }\end{array}$ & $\begin{array}{l}\text { Mawalla et } \\
\text { al } 2011 \text { ( } \\
\text { 29) }\end{array}$ \\
\hline 20 & $27.3 \%(6)$ & $16.7 \%(1)$ & $\begin{array}{l}\text { Woman aged } 14 \text { to } 44 \\
\text { who have developed } \\
\text { surgical site } \\
\text { infections after } \\
\text { having undergone a } \\
\text { caesarean section. } \\
345 \text { woman were } \\
\text { swabbed between } \\
\text { October } 2011 \text { and } \\
\text { February } 2012\end{array}$ & $\begin{array}{l}\text { Surgical site } \\
\text { wound } \\
\text { infection }\end{array}$ & $\begin{array}{l}\text { Mwanza } \\
\text { (Lake } \\
\text { zone) }\end{array}$ & $\begin{array}{l}\text { Mpogoro } \\
\text { et al } \\
2014 \text { ( } 30)\end{array}$ \\
\hline
\end{tabular}

\section{Antibiotic resistance in human originated isolates.}

The primary method for establishing $S$. aureus antibiogram in the reviewed work was done phenotypically by the use of the Kirby-Bauer disk diffusion test along with Clinical Laboratory Standard Institute (CLSI) guidelines. Kirby-Bauer is a test for antimicrobial susceptibility of bacteria, based on the size of inhibition zones of bacterial growth in a lawn culture around disks impregnated with the antimicrobial drug (44). This method has been reported to have inherent shortcomings such as being highly dependent on experimental conditions that may affect end results (45). With this fact in mind the Kirby Bauer antibiogram results would have to be confirmed by another test of a different principle such as genomic based method to prevent over reporting of resistance, particularly in the confirmation of MRSA and VRSA 


\begin{tabular}{|c|c|c|c|c|c|c|}
\hline S.No & $\begin{array}{l}\text { Prevalence } \\
\text { S. aureus \% } \\
\text { (n) }\end{array}$ & $\begin{array}{l}\text { Prevalence } \\
\text { MRSA \% } \\
\text { (n) }\end{array}$ & Population & $\begin{array}{l}\text { Source of } \\
S \\
\text { aureus isolates }\end{array}$ & Location & Reference \\
\hline 21 & $29.2 \%(7)$ & NR & $\begin{array}{l}162 \text { patients of all age } \\
\text { group and gender who } \\
\text { underwent major limb } \\
\text { amputations at BMC } \\
\text { between March } 2008 \text { and } \\
\text { February 2010. } 24 \text { of the } \\
\text { participants had surgical } \\
\text { site infections out of which } \\
\text { different bacteria were } \\
\text { recovered as the cause of } \\
\text { infection. }\end{array}$ & $\begin{array}{l}\text { Surgical } \\
\text { wound } \\
\text { infection }\end{array}$ & $\begin{array}{l}\text { Mwanza } \\
\text { (Lake } \\
\text { zone) }\end{array}$ & $\begin{array}{l}\text { Chalya et } \\
\text { al } 2012 \text { ( } \\
31 \text { ) }\end{array}$ \\
\hline 22 & $59.3 \%$ & NR & $\begin{array}{l}\text { All patients of all age } \\
\text { groups and gender } \\
\text { presenting with animal } \\
\text { related injuries at the BMC } \\
\text { between September } 2007 \\
\text { and August } 2011 \text {. } \\
\text { Postoperative wound } \\
\text { infection was the most } \\
\text { commonest complication } \\
\text { reported lead by } S \text { aureus } \\
\text { infections }\end{array}$ & $\begin{array}{l}\text { Surgical site } \\
\text { wound } \\
\text { infections }\end{array}$ & $\begin{array}{l}\text { Mwanza } \\
\text { (Lake } \\
\text { zone) }\end{array}$ & $\begin{array}{l}\text { Gilyoma } \\
\text { et al } \\
2013( \\
32)\end{array}$ \\
\hline 23 & $\begin{array}{l}\text { Overall } \\
\text { prevalence } \\
16.1 \text { (25) out } \\
\text { of which } 28 \% \\
\text { (7) were } \\
\text { isolated in } \\
\text { HIV positive } \\
\text { patients and } \\
72 \%(18) \text { in } \\
\text { HIV negative } \\
\text { patients }\end{array}$ & NR & $\begin{array}{l}\text { The study was done to } \\
\text { compare magnitude of } \\
\text { bacterial resistance to } \\
\text { cotrimoxazole/other } \\
\text { antimicrobials among } \\
\text { isolates from HIV infected } \\
\text { patients on cotrimoxazole } \\
\text { prophylaxis and those not } \\
\text { on prophylaxis and non- } \\
\text { HIV patients attending } \\
\text { BMC between January and } \\
\text { October } 2012\end{array}$ & $\begin{array}{l}\text { Urine and } \\
\text { wound swabs }\end{array}$ & $\begin{array}{l}\text { Mwanza } \\
\text { (Lake } \\
\text { zone) }\end{array}$ & $\begin{array}{l}\text { Marwa et } \\
\text { al } 2015 \text { ( } \\
\text { 33) }\end{array}$ \\
\hline 24 & $18.2 \%(34)$ & $41.2 \%(14)$ & $\begin{array}{l}301 \text { patients aged more } \\
\text { than one year who } \\
\text { presented with ear } \\
\text { discharge for more than } 6 \\
\text { weeks and tympanic } \\
\text { membrane perforation at } \\
\text { the ENT department } \\
\text { between October } 2013 \text { and } \\
\text { March } 2014 \text { were recruited } \\
\text { into the study }\end{array}$ & Ear infection & $\begin{array}{l}\text { Mwanza } \\
\text { (Lake } \\
\text { zone) }\end{array}$ & $\begin{array}{l}\text { Mushi et } \\
\text { al } 2016 \text { ( } \\
34)\end{array}$ \\
\hline 25 & $\begin{array}{l}\text { Overall } \\
22.8 \%(100) \\
\text { Blood } 80 \% \\
(80) \\
\text { Pus swabs } \\
18 \%(18)\end{array}$ & $\begin{array}{l}\text { Only } 78 \\
\text { were } \\
\text { subjected } \\
\text { to } \\
\text { AST.34.6\% }\end{array}$ & $\begin{array}{l}\text { A total of } 3330 \\
\text { microbiological culture } \\
\text { results scripts } \\
\text { representing non-repetitive } \\
\text { specimens reported } \\
\text { between June } 2013 \text { and }\end{array}$ & $\begin{array}{l}\text { Bacteremia, } \\
\text { wound } \\
\text { infection, } \\
\text { urinary tract } \\
\text { infection and }\end{array}$ & $\begin{array}{l}\text { Mwanza } \\
\text { (Lake } \\
\text { zone) }\end{array}$ & $\begin{array}{l}\text { Moremi et } \\
\text { al } 2016 \text { ( } \\
35 \text { ) }\end{array}$ \\
\hline
\end{tabular}

Page 10/30 


\begin{tabular}{|c|c|c|c|c|c|c|}
\hline & $\begin{array}{l}\text { Other } \\
\text { infections } 2 \% \\
(2)\end{array}$ & $\begin{array}{l}\text { (27) were } \\
\text { MRSA }\end{array}$ & $\begin{array}{l}\text { May } 2015 \text { were retrieved } \\
\text { and analyzed for } \\
\text { pathogens and their } \\
\text { susceptibility patterns } \\
\text { using STATA-11 software. }\end{array}$ & $\begin{array}{l}\text { pulmonary } \\
\text { infection }\end{array}$ & & \\
\hline 26 & $55.3 \%(68)$ & NR & $\begin{array}{l}\text { Primary school children } \\
\text { (aged 6-15) from } 4 \text { schools } \\
\text { in Moshi municipality } \\
\text { assessed/self-reported } \\
\text { respiratory tract infection } \\
\text { symptoms. The community } \\
\text { based study was conducted } \\
\text { between January and } \\
\text { March } 2014 \text {. }\end{array}$ & $\begin{array}{l}\text { Nasal and } \\
\text { Trought swabs }\end{array}$ & $\begin{array}{l}\text { Moshi } \\
\text { Northen } \\
\text { zone }\end{array}$ & $\begin{array}{l}\text { Ngocho et } \\
\text { al } 2015 \text { ( } \\
\text { 36) }\end{array}$ \\
\hline 27 & $\begin{array}{l}66 \%(103) \text { at } \\
6 \text { weeks, } \\
36 \%(47) \text { at } \\
3 \text { months and } \\
24 \% \text { (33) at } \\
6 \text { months. } \\
38 \% \text { (17) } \\
\text { mothers } \\
\text { were } \\
\text { colonized by } \\
\text { S aureus } \\
\text { parallel to } \\
\text { their } \\
\text { children. }\end{array}$ & NR & $\begin{array}{l}\text { Children born to HIV } \\
\text { positive mothers attending } \\
\text { RCH clinics to establish } \\
\text { prevalence and influence } \\
\text { of nasal pharyngeal } \\
\text { bacterial colonization on } \\
\text { children. Nasal swabs from } \\
\text { children were taken at } 6 \\
\text { weeks ( } \mathrm{n}=156) \text { ), } 3 \text { (n=136) } \\
\text { and } 6 \text { ( }=130 \text { ) months } \\
\text { consecutively. Mothers } \\
\text { (45) of the infants were } \\
\text { also swabbed at } 3 \text { and } 6 \\
\text { month visits ( Study } \\
\text { duration 2005-2009 }\end{array}$ & Nasal carriage & $\begin{array}{l}\text { Moshi } \\
\text { Northen } \\
\text { zone }\end{array}$ & $\begin{array}{l}\text { Kinabo et } \\
\text { al } 2013 \text { ( } \\
37 \text { ) }\end{array}$ \\
\hline 28 & $16 \%(23)$ & NR & $\begin{array}{l}\text { Patients presenting with } \\
\text { SSI, infected diabetic } \\
\text { wounds, infected wounds } \\
\text { due to trauma, and } \\
\text { patients with other } \\
\text { infected wounds admitted } \\
\text { in surgical } \\
\text { ward (study duration July } \\
2013 \text { to June 2014) }\end{array}$ & $\begin{array}{l}\text { Wound } \\
\text { infections }\end{array}$ & $\begin{array}{l}\text { Moshi } \\
\text { (Northen } \\
\text { zone) }\end{array}$ & $\begin{array}{l}\text { Kassam } \\
\text { et al } \\
2017( \\
38)\end{array}$ \\
\hline 29 & $\begin{array}{l}9.3 \% \text { (35) of } \\
\text { which } 82.9 \\
\text { (29) were } \\
\text { from wound } \\
\text { infections, } \\
11 \% \text { (4) from } \\
\text { blood } \\
\text { samples and } \\
6 \%(2) \text { from } \\
\text { sputum } \\
\text { samples }\end{array}$ & $\begin{array}{l}\text { Only } 22 \\
\text { isolates } \\
\text { were } \\
\text { subjected } \\
\text { to AST. } \\
27.3 \% \text { (6) } \\
\text { were } \\
\text { MRSA }\end{array}$ & $\begin{array}{l}\text { People admitted to the } \\
\text { medical or surgical wards } \\
\text { at KCMC between } 2013 \\
\text { and } 2015 \text {. The study } \\
\text { collected stool, sputum, } \\
\text { blood and wound/pus } \\
\text { samples from patients of } \\
\text { all age groups to describe } \\
\text { causative agents of } \\
\text { different infections. }\end{array}$ & $\begin{array}{l}\text { Wound } \\
\text { infection } \\
\text { Blood } \\
\text { infection } \\
\text { Bronchial } \\
\text { infection }\end{array}$ & $\begin{array}{l}\text { Moshi } \\
\text { (Northen } \\
\text { zone) }\end{array}$ & $\begin{array}{l}\text { Kumburu } \\
\text { et al } \\
\text { 2017 ( } \\
\text { 39) }\end{array}$ \\
\hline 30 & $9.1 \%(6)$ & NR & $\begin{array}{l}\text { A total of } 867 \text { patients } \\
\text { aged between } 2 \text { and } 5 \text { with } \\
\text { fever above } 37^{0} \mathrm{C} \text { were }\end{array}$ & $\begin{array}{l}\text { Urinary tract } \\
\text { infection }\end{array}$ & $\begin{array}{l}\text { Tanga } \\
\text { (Korogwe) }\end{array}$ & $\begin{array}{l}\text { Mahende } \\
\text { et al }\end{array}$ \\
\hline
\end{tabular}

Page $11 / 30$ 


\begin{tabular}{|c|c|c|c|c|c|c|}
\hline & & & $\begin{array}{l}\text { enrolled between January } \\
\text { and October } 2013.373 \\
\text { urine samples were } \\
\text { collected and } 66 \text { tested } \\
\text { positive for UTI. All S } \\
\text { aureus isolates were } \\
\text { recovered from UTI } \\
\text { patients }\end{array}$ & & $\begin{array}{l}\text { (Northen } \\
\text { zone) }\end{array}$ & $\begin{array}{l}2014( \\
40)\end{array}$ \\
\hline 31 & $17 \%(17)$ & NR & $\begin{array}{l}\text { Children aged 0-12yrs } \\
\text { dismissed from the } \\
\text { hospital with pneumonia } \\
\text { diagnosis.100 children } \\
\text { were enrolled in the } \\
\text { attempt to characterize } \\
\text { etiology of community } \\
\text { acquired pneumonia. } \\
\text { (study duration August } \\
\text { 2014- April 2015) }\end{array}$ & Blood infection & $\begin{array}{l}\text { Itigi } \\
\text { (Central } \\
\text { zone) }\end{array}$ & $\begin{array}{l}\text { Caggiano } \\
\text { et al } \\
2017(41)\end{array}$ \\
\hline 32 & $11.3 \%(9)$ & $0 \%$ & $\begin{array}{l}\text { Febrile adults and children } \\
\text { seeking care at the Mnazi } \\
\text { mmoja hospital between } \\
\text { March 2012- April } 2013\end{array}$ & Blood infection & Zanzibar & $\begin{array}{l}\text { Onken et } \\
\text { al } 2015 \text { ( } \\
42)\end{array}$ \\
\hline 33 & $6.3 \%(5)$ & $0 \%$ & $\begin{array}{l}\text { Febrile patients seeking } \\
\text { outpatient health care at } 3 \\
\text { different district hospitals } \\
\text { (Wete, Chake chake, and } \\
\text { Mkoani) on Pemba island } \\
\text { between March } 2009 \text { to } \\
\text { December } 2010\end{array}$ & Blood infection & Zanzibar & $\begin{array}{l}\text { Thriemer } \\
\text { et al } \\
2012( \\
43)\end{array}$ \\
\hline
\end{tabular}

strains. In this review, four studies had MRSA confirmed genotypically which showed $100 \%$ agreement with the phenotypic Kirby-Bauer disk diffusion test. This agreement between the methods should encourage the use of the Kirby-Bauer disk diffusion test as an antibiotic sensitivity monitoring strategy in the country, since it is the most available and affordable method in our settings.

Twenty two (22) studies contributed information on antibiotic susceptibility of $S$. aureus bacteria. MRSA in most cases was identified by resistance against cefoxitin, however one study used methicillin as their identification drug. As summarized in Table 3, extremely high resistance rates against $\beta$-lactams i.e. penicillin (87 - 99\%) and ampicillin (67-91\%) were observed.

S. aureus colonization is considered being a risk factor for eventual infection as well as a potential source of spread of resistant strains. Examination of colonization strains is therefore key in understanding the epidemiology of $S$. aureus infection/diseases. This review discovered that the antibiotic resistance patterns in colonization strains showed uncharacteristically high resistance to commonly used antibiotics compared to studies reported in Europe and Asia. In den Heijer et al 2013's report about antibiotic resistance of commensal S. aureus across Europe described resistance rates lower than $20 \%$ against erythromycin, gentamicin, tetracycline and co-trimoxazole (46). Similar low resistance rates were reported in some parts of Asia with an exception of tetracycline which was slightly higher than 30\% (47). 
This review on the other hand recorded higher mean resistance rates of $49 \%, 38 \%$ and $28 \%$ against erythromycin, co-trimoxazole and tetracycline respectively. Further resistance against gentamicin was significantly higher than recorded in other parts outside Africa $(46,47)$.

Resistance pattern in clinical $S$. aureus observed in this review were in accordance with other reports around Africa $(48,49)$. As in many parts recorded resistance against $\beta$ lactams was high, further markedly resistance rates ranging from $44-50 \%$ against erythromycin, gentamicin, co-trimoxazole and clindamycin were also described in this review. The affected antibiotics are readily available in the community and also commonly used for empirically treatment of different cases of bacterial suspected infections. A notable presence of resistance against these antibiotics is an indication of their failure in treatment and a need for broader class antibiotics which are expensive and not accessible for most Tanzanians.

MRSA prevalence reported in different studies in this review was between 19-33\% which seems to have risen in the last 10 years. Abdulgader et al 2015 in the review of MRSA prevalence on the African continent categorized Tanzania as belonging to countries with low MRSA prevalence ranging from $6-16 \%$ between the years 2001-2009 (50). This abrupt increase should be taken seriously by which more research efforts should be directed towards understanding and preventing further spread of these strains in order to safe guard much needed antibiotics

Table 3: Antimicrobial resistance rates of $S$. aureus in humans 


\begin{tabular}{|c|c|c|}
\hline Antibiotic ( $\left.N^{*}\right)$ & $\begin{array}{l}\text { Resistance rates in } S . \text { aureus isolates from humans colonization. } \\
\text { References (\% range) }\end{array}$ & $\begin{array}{l}\text { Mean resistance } \\
\left(n / N^{*}\right)(\%)\end{array}$ \\
\hline Penicillin(267) & $(11-13)$ & $(263 / 267) \quad(99 \%)$ \\
\hline $\begin{array}{l}\text { Ampicillin } \\
(151)\end{array}$ & $(17,24,36)$ & $\begin{array}{l}(133 / 151) \\
(88 \%)\end{array}$ \\
\hline $\begin{array}{l}\text { Tetracycline } \\
(223)\end{array}$ & $(12,14,17,24)$ & $\begin{array}{l}(63 / 223) \\
(28 \%)\end{array}$ \\
\hline $\begin{array}{l}\text { Erythromycin } \\
(244)\end{array}$ & $(11,12,24)$ & $\begin{array}{l}(119 / 244) \\
(49 \%)\end{array}$ \\
\hline $\begin{array}{l}\text { Gentamicin } \\
(382)\end{array}$ & $(11,13,14,17,36)$ & $\begin{array}{l}(99 / 382) \\
(26 \%)\end{array}$ \\
\hline $\begin{array}{l}\text { Co-trimoxazole } \\
(314)\end{array}$ & $(11,12,14,17)$ & $\begin{array}{l}(118 / 314) \\
(38 \%)\end{array}$ \\
\hline $\begin{array}{l}\text { Ciprofloxacin } \\
\text { (233) }\end{array}$ & $(13,14)$ & $(15 / 233)$ \\
\hline $\begin{array}{l}\text { Clindamycin } \\
(178)\end{array}$ & $(11,12)$ & $\begin{array}{l}(30 / 178) \\
(14 \%)\end{array}$ \\
\hline \multirow[t]{2}{*}{ Cefoxitin (388) } & $(11,12,14,24)$ & $\begin{array}{l}(93 / 388) \\
(24 \%)\end{array}$ \\
\hline & $\begin{array}{l}\text { Resistance rates in } S . \text { aureus isolates from invasive (blood-born) infections. } \\
\text { References (\% range) }\end{array}$ & \\
\hline Penicillin (46) & $(25,42,43)$ & $\begin{array}{l}(40 / 46) \\
(87 \%)\end{array}$ \\
\hline Ampicillin (27) & $(20)$ & $\begin{array}{l}(23 / 27) \\
(85 \%)\end{array}$ \\
\hline $\begin{array}{l}\text { Erythromycin } \\
\text { (37) }\end{array}$ & $(25,43)$ & $\begin{array}{l}(23 / 37) \\
(62 \%)\end{array}$ \\
\hline $\begin{array}{l}\text { Gentamicin } \\
(27)\end{array}$ & $(20)$ & $\begin{array}{l}(15 / 27) \\
(56 \%)\end{array}$ \\
\hline $\begin{array}{l}\text { Co-trimoxazole } \\
\text { (46) }\end{array}$ & $(25,42,43)$ & $\begin{array}{l}(23 / 46) \\
(50 \%)\end{array}$ \\
\hline
\end{tabular}




\begin{tabular}{|c|c|c|c|}
\hline $\begin{array}{l}\text { Clindamycin } \\
\text { (32) }\end{array}$ & $(25)$ & (44) & $\begin{array}{l}(14 / 32) \\
(44 \%)\end{array}$ \\
\hline $\begin{array}{l}\text { Methicillin } \\
(245)\end{array}$ & $(18)$ & (23) & $\begin{array}{l}(57 / 245) \\
(23 \%)\end{array}$ \\
\hline \multirow[t]{2}{*}{ Cefoxitin (46) } & $(25,42,43)$ & $(0-32)$ & $\begin{array}{l}(9 / 46) \\
(20 \%)\end{array}$ \\
\hline & $\begin{array}{l}\text { Resistance rates in } S \\
\text { References (\% range) }\end{array}$ & aureus isolates from non-invasive infections. & \\
\hline Penicillin (202) & $(21,22,28,33,51)$ & $(30-100)$ & $\begin{array}{l}(183 / 202) \\
(91 \%)\end{array}$ \\
\hline $\begin{array}{l}\text { Ampicillin } \\
(263)\end{array}$ & $(20,23)$ & $(47-88)$ & $\begin{array}{l}(177 / 263) \\
(67 \%)\end{array}$ \\
\hline $\begin{array}{l}\text { Tetracyclin } \\
(67)\end{array}$ & $(33,34,51)$ & $(6-45)$ & $\begin{array}{l}(14 / 67) \\
(21 \%)\end{array}$ \\
\hline $\begin{array}{l}\text { Erythromycin } \\
(221)\end{array}$ & $(28,33,34,51)$ & $(14-46)$ & $\begin{array}{l}(81 / 221) \\
(37 \%)\end{array}$ \\
\hline $\begin{array}{l}\text { Gentamicin } \\
\text { (339) }\end{array}$ & $(20,23,28,34,51)$ & $(11-52)$ & $\begin{array}{l}(110 / 339) \\
(32 \%)\end{array}$ \\
\hline $\begin{array}{l}\text { Co-trimoxazole } \\
(232)\end{array}$ & $(23,28,33,34,51)$ & $(43-74)$ & $\begin{array}{l}(115 / 232) \\
(50 \%)\end{array}$ \\
\hline $\begin{array}{l}\text { Ciprofloxacin } \\
\text { (187) }\end{array}$ & $(23,33,34)$ & $(4-14)$ & $\begin{array}{l}(20 / 187) \\
(17 \%)\end{array}$ \\
\hline $\begin{array}{l}\text { Clindamycin } \\
(42)\end{array}$ & $(28,51)$ & $(21-63)$ & $\begin{array}{l}(19 / 42) \\
(45 \%)\end{array}$ \\
\hline Cefoxitin (226) & $(21,22,28,34,51)$ & $(1-100)$ & $\begin{array}{l}(55 / 226) \\
(24 \%)\end{array}$ \\
\hline
\end{tabular}

\section{Genotypic characterisation of human originated $S$. aureus isolates}

Four human related studies were reviewed to inform this section (summarized in Table 4). A wide spectrum of MSSA spa types and sequence types (ST) were identified in the review. Nevertheless the limited number of publications on genotypic data could not reveal dominance in any particular strain. 
Two publications gave an account on genetic characterization of $\operatorname{MRSA}(39,51)$. The observed MRSA were dominated by ST88, however sequence types ST8, ST1719, ST 1820, and ST239 were also reported. None of the MRSA were $p v /$ positive consistent with the findings by Abdulgader et al 2015 who suggested that Africa is predominated by $p v /$ negative MRSA belonging to ST88 (50).

Even though some important information was apparent in this review data concerning MSSA and MRSA genotyping is still limited, Kumburu et al 2018 for example was the only one who reported on other virulence factors apart from $\mathrm{pv} /$ hence still lacking knowledge on other important factors associated to the bacteria circulating in our communities. Furthermore genotypic characterization studies predominantly focused on screening for the $m e c A$ gene the $\beta$-lactam associated resistance gene, neglecting other resistant markers for other commonly used antibiotic in the country. Nurjadi et al 2014 whilst researching on trimethoprim resistance genes in $S$. aureus isolated from different countries determined that $100 \%$ of the Tanzanian isolated in the study which were phenotypically trimethoprim resistant in fact harboured a trimethoprim conferring resistant gene (52). This emphasises the need for research efforts to be directed towards researching a wider range of resistance causing genes in $S$. aureus to get comprehensive resistance profile data.

Most $p v /$ positive MSSA clones associate with skin infections identified in this review belonged to ST152 which concurs with the findings from other African based studies (53).

\section{Table 4: $S$. aureus genetic diversity as described by the reviewed publications}




\begin{tabular}{|c|c|c|c|c|c|c|}
\hline Author & Host & Sample type & $\begin{array}{l}\text { Resistant } \\
\text { gene }\end{array}$ & $\begin{array}{l}\text { Virulence } \\
\text { factor }\end{array}$ & spa Type & MLST \\
\hline $\begin{array}{l}\text { Moremi et } \\
\text { al } 2012 \text { ( } \\
51)\end{array}$ & Human & $\begin{array}{l}\text { Wound, pus, } \\
\text { nasal swabs ( } 24 \\
\text { MRSA strains } \\
\text { from previous } \\
\text { studies) }\end{array}$ & $\operatorname{mec} A(100 \%)$ & pvl (16.7\%) & $\begin{array}{l}\text { t7231, t690 } \\
\text { t064, t104, } \\
\text { t1855,t7237 } \\
\text { t186, } \\
\text { t667 }\end{array}$ & $\begin{array}{l}\text { ST88, } \\
\text { ST1719, } \\
\text { ST8,ST1820 }\end{array}$ \\
\hline $\begin{array}{l}\text { Kumburu } \\
\text { et al } \\
2018 \text { ( } 39 \text { ) }\end{array}$ & Human & $\begin{array}{l}\text { Blood, Sputum, } \\
\text { wound/pus }\end{array}$ & mecA (33.3\%) & $\begin{array}{l}\text { pvl }(16.7 \%) \\
\text { txt }(6.7 \%) \\
\text { both in non } \\
\text { mecA isolates }\end{array}$ & ND & $\begin{array}{lr}\text { ST-8, } & \text { ST-1, } \\
\text { 152, } & \text { ST1719, } \\
\text { ST15, } & \text { ST1847, } \\
\text { ST188, } & \text { ST22, } \\
\text { ST239, } & \text { ST30, } \\
\text { ST5, ST580, ST6, } \\
\text { ST97 }\end{array}$ \\
\hline $\begin{array}{l}\text { Katakweba } \\
\text { et al } \\
2016 \text { ( } 17)\end{array}$ & Human & Nasal swabs & $\begin{array}{l}\text { mecA and } \\
\text { mecC } \\
\text { (None } \\
\text { detected) }\end{array}$ & NS & $\begin{array}{lr}\text { t714, } & \text { t148, } \\
\text { t084, } & \text { t002, } \\
\text { t223, } & \text { t314, } \\
\text { t311, } & \text { t015, } \\
\text { t451, } & \text { t690, } \\
\text { t1849, } & \text { t2030, } \\
\text { t10779* }\end{array}$ & $\begin{array}{l}\text { ST15, ST18, } \\
\text { ST72, ST22, ST5, } \\
\text { ST121, ST231, } \\
\text { ST45, ST8 }\end{array}$ \\
\hline $\begin{array}{l}\text { Katakweba } \\
\text { et al } \\
2016 \text { ( } 17)\end{array}$ & Pig & Nasal swab & $\begin{array}{l}\operatorname{mecA} \text { and } \\
\operatorname{mec} C \\
\text { (None } \\
\text { detected) }\end{array}$ & NS & t131 & ST80 \\
\hline $\begin{array}{l}\text { Katakweba } \\
\text { et al } \\
2016 \text { ( 17) }\end{array}$ & Dog & Nasal swabs & $\begin{array}{l}\text { mecA and } \\
\text { mecC } \\
\text { (None } \\
\text { detected) }\end{array}$ & NS & $\begin{array}{l}\text { t084, } t 127, \\
\text { t223, t314, } \\
\text { t267, t508, } \\
\text { t1476 }\end{array}$ & $\begin{array}{l}\text { ST15,ST18, ST1, } \\
\text { ST22, ST121, }\end{array}$ \\
\hline $\begin{array}{l}\text { Nurjadi et } \\
\text { al } 2014 \text { ( } \\
52)\end{array}$ & Human & $\begin{array}{l}\text { Nasal, } \quad \text { SSTI } \\
\text { swabs }\end{array}$ & $\begin{array}{l}\text { dfrA, } \quad d f r G \\
\text { and dfrK. }\end{array}$ & NS & ND & ND \\
\hline
\end{tabular}




\begin{tabular}{|l|l|l|l|}
$\mid \begin{array}{l}\text { ffrG detected } \\
\text { by } 37 \% *\end{array}$ & & \\
\hline
\end{tabular}

ND: Not done, NS: Not screened

\section{Prevalence, antibiotic resistance and genotyping of $S$. aureus isolated from animals.}

Studies reporting on prevalence, antimicrobial resistance and genetic characteristics of $S$ aureus in animals published between 2010 and 2018 in Tanzania were few. Only 5 studies were included in this review, scantly representing a small area in the country. Three publications reported on $S$. aureus prevalence in milk samples, one study described prevalence in milk and cow nasal swabs and the last study reported on $S$. aureus prevalence in pig and dog nasal swabs.

\section{Prevalence of $S$ aureus in Animals}

As indicated in table 5 most examined specimen in animals was milk. Prevalence of $S$. aureus varied in different geographic area. Kashoma et al 2018 and Mohammed et al 2018 reported the highest prevalence at $49 \%$ and $41 \%$ in Morogoro respectively $(54,55)$. Suleiman et al $2018(56)$ did not fall far back when observing a prevalence of $37 \%$ describing subclinical mastitis in cows on Unguja Island. The lowest prevalence of $33 \%$ was recorded in a study aiming at assessing raw milk quality in Arusha and Meru district(57). Generally prevalence of $S$. aureus seems to be much lower in swabs taken from nasal cavities as shown by Kashoma et al 2018 who swabbed cows observed a prevalence of $28 \%$ whereas Katakweba et al 2016(17) observed a prevalence of $4 \%$ and $11 \%$ in pig and dog nasal swabs respectively.

MRSA detection in milk associated studies varied tremendously ranging from 4- $35 \%(54,55)$, even though both studies reporting on this data were conducted in Morogoro township. Furthermore, Kashoma et al 2018 reported MRSA prevalence of $16 \%$ isolates recovered from cow nasal cavities, whereas no MRSA was detected by Katakweba et al 2016 in pigs and dogs nasal swabs.

\section{Table 5: $S$. aureus prevalence in Animal related samples}




\begin{tabular}{|c|c|c|c|c|c|c|}
\hline S.No & $\begin{array}{l}\text { Prevalence } \\
\text { S aureus \% } \\
\text { (n) }\end{array}$ & $\begin{array}{l}\text { Prevalence } \\
\text { MRSA \% } \\
\text { (n) }\end{array}$ & Population & $\begin{array}{l}\text { Source of } \\
S \\
\text { aureus isolates }\end{array}$ & Location & Reference \\
\hline 1 & $\begin{array}{l}\text { Milk: } 49 \% \\
\text { (49) } \\
\text { Cow nasal } \\
\text { swabs : } \\
28 \% \text { (37) }\end{array}$ & $\begin{array}{l}\text { Milk: 35\% } \\
\text { (17) } \\
\text { Cow nasal } \\
\text { swabs: } \\
16 \%(6)\end{array}$ & $\begin{array}{l}\text { The study was carried out in } 3 \text { dairy } \\
\text { farms (A, B and C) that belonged to } \\
\text { Sokoine University of Agriculture. All } \\
\text { farms were located within the same } \\
\text { climatic zone. All } 3 \text { farms carried out } \\
\text { mixed animal farming }\end{array}$ & $\begin{array}{l}\text { Milk samples } \\
\text { and cow nasal } \\
\text { swabs }\end{array}$ & $\begin{array}{l}\text { Morogoro } \\
\text { (Eastern } \\
\text { zone) }\end{array}$ & $\begin{array}{l}\text { Kashoma et } \\
\text { al } 2018 \text { ( } \\
55 \text { ) }\end{array}$ \\
\hline 2 & $41 \%(48)$ & $4 \%(3)$ & $\begin{array}{l}\text { The study involved } 18 \text { of the } 29 \\
\text { Wards within Morogoro Municipality. } \\
\text { In each of the selected Wards, sales } \\
\text { points and local shops where raw } \\
\text { milk is sold were randomly selected. } \\
\text { A total of } 117 \text { milk samples. The } \\
\text { study was conducted between } \\
\text { January and June } 2015\end{array}$ & Milk samples & $\begin{array}{l}\text { Morogoro } \\
\text { (Eastern } \\
\text { zone) }\end{array}$ & $\begin{array}{l}\text { Mohammed } \\
\text { et al } \\
2018 \text { ( 54) }\end{array}$ \\
\hline 3 & $37 \%(217)$ & NR & $\begin{array}{l}\text { Study conducted between January - } \\
\text { July } 2014 \text { on Unguja Island to } \\
\text { establish prevalence of subclinical } \\
\text { mastitis in smallholder dairy cows } \\
\text { and pattern of antimicrobial } \\
\text { susceptibility of major mastitis } \\
\text { pathogens. }\end{array}$ & Milk samples & $\begin{array}{l}\text { Unguja } \\
\text { (Zanzibar) }\end{array}$ & $\begin{array}{l}\text { Suleiman } \\
\text { et al } \\
2018 \text { ( 56) }\end{array}$ \\
\hline 4 & $33 \%(30)$ & NR & $\begin{array}{l}105 \text { milk samples from smallholder } \\
\text { dairy farmers, milk vendors and } \\
\text { retailers were taken within Arusha } \\
\text { City and Meru District to assess the } \\
\text { quality of raw milk and stakeholders' } \\
\text { awareness on milk-borne health risks } \\
\text { and factors for poor milk hygiene. }\end{array}$ & Milk samples & $\begin{array}{l}\text { Arusha } \\
\text { city and } \\
\text { Meru } \\
\text { district } \\
\text { (Northern } \\
\text { zone) }\end{array}$ & $\begin{array}{l}\text { Ngasala et } \\
\text { al } 2015 \text { ( } \\
57)\end{array}$ \\
\hline 5 & $\begin{array}{l}\text { Pig nasal } \\
\text { swabs } 4 \% \\
(4) \\
\text { Dog nasal } \\
\text { swabs } 11 \% \\
(11)\end{array}$ & $\begin{array}{l}\text { Pig swabs: } \\
0 \% \\
\text { Dog nasal } \\
\text { swabs: } 0 \%\end{array}$ & $\begin{array}{l}100 \text { pigs and } 100 \text { dogs dwelling in } \\
\text { urban and peri urban Morogoro } \\
\text { Municipality }\end{array}$ & Nasal swabs & $\begin{array}{l}\text { Morogoro } \\
\text { (Eastern } \\
\text { zone) }\end{array}$ & $\begin{array}{l}\text { Katakweba } \\
\text { et al } \\
2016 \text { ( 17) }\end{array}$ \\
\hline
\end{tabular}

NR: Not reported

Table 6: Antibiotic resistance in animals 


\begin{tabular}{|c|c|c|c|c|}
\hline \multirow{2}{*}{$\begin{array}{l}\left.\text { Antibiotic ( } \boldsymbol{N}^{*}\right) \\
\text { Penicillin(265) }\end{array}$} & \multicolumn{2}{|c|}{$\begin{array}{l}\text { Resistance rates in different studies in Animals. } \\
\text { (References) (range\%) }\end{array}$} & \multicolumn{2}{|c|}{ Mean resistance $\left(n / N^{*}\right)(\%)$} \\
\hline & $(54,56)$ & $(72-88)$ & $(225 / 265)$ & $(85 \%)$ \\
\hline Ampicillin (99) & $(17,55)$ & $(62-100)$ & $(72 / 99)$ & $(73 \%)$ \\
\hline Tetracycline (364) & $(17,54-56)$ & $(16-73)$ & $(110 / 364)$ & $(30 \%)$ \\
\hline Erythromycin (301) & $(55,56)$ & $(5-23)$ & $(28 / 301)$ & $(9 \%)$ \\
\hline Gentamicin (99) & $(17,55)$ & $(6-25)$ & $(4 / 99)$ & $(4 \%)$ \\
\hline Co-trimoxazole (132) & $(54,55)$ & $(22-40)$ & $(42 / 132)$ & $(32 \%)$ \\
\hline Vancomycin (132) & $(17,54,55)$ & $(2-11)$ & $(10 / 132)$ & $(8 \%)$ \\
\hline Amoxicillin (217)* & $(56)$ & (47) & $(102 / 217)$ & $(47 \%)$ \\
\hline Cephalexin (217)* & $(56)$ & (30) & $(64 / 217)$ & $(30 \%)$ \\
\hline Oxacillin (147) & $(17,54,55)$ & $(0-34)$ & $(25 / 147)$ & $(17 \%)$ \\
\hline Cefoxitin (132) & $(54,55)$ & $(4-17)$ & $(13 / 132)$ & $(10 \%)$ \\
\hline
\end{tabular}

*Reported in one study only

\section{Antibiotic resistance in animal originated $S$ aureus isolates}

Four studies described resistance in S. aureus using different antibiotic pallets which included cephalexin, gentamicin, kanamycin, neomycin, tetracycline, streptomycin, amoxicillin, cephalexin, clindamycin, vancomycin, ampicillin, co-trimoxazole, oxacillin, and cefotixin. This review however focused on the most reported antibiotics in this area as indicated in Table 6.

Most of the reviewed studies reported low antibiotic resistance levels with exception of penicillin and ampicillin which showed mean resistances rates of $85 \%$ and $73 \%$ respectively. Suleiman et al 2018 also further observed resistance against amoxicillin and cephalexin at a rate of $47 \%$ and $30 \%$, which was unfortunately not reported by any of the other reviewed studies. Oxy-tetracycline has been stated as being the most over used antibiotic in the livestock keeping business in Tanzania (58). The mean resistance rates against oxacillin (17\%) and tetracycline (30\%) observed in this review are indicative of the effects of previously reported overuse.

MRSA detection in all studies was confirmed by resistance against cefoxitin and/or oxacillin, whereby mean resistance rates of $10 \%$ and $17 \%$ were observed respectively. An average of $8 \%$ resistance against vancomycin was observed, reported in $3 / 5$ studies as described in 
Table 6.

\section{Genotypic characterisation of animal originated S. aureus isolates}

Genotypic data were retrieved from 2 of the 5 studies reviewed. In both, real time- PCR (qPCR) was used to confirm S. aureus as well as screening for $m e c A$ resistance conferring gene. Katakweba et al 2016 additionally screened for $m e c C$ a recently discovered mecA homologue said to have the potential to be mis-categorized as Methicillin sensitive $S$. aureus (MSSA). None of the two resistance conferring genes were detected in Katakweba's study, however $S$. aureus was confirmed in 4 pigs and 11 dog samples respectively. Further spa typing of the pig originating MSSA characterized them as spa type t131 associated with ST80. Additionally isolates of dog origin were characterized as spa types t314 (ST121), t084 (ST15 and ST18) and t223 (ST22) all of which were also identified in human nasal isolates reported in the same study.

In the study by Mohammed et al 2018 , both coagulase positive $(n=46)$ and coagulase negative $(n=2) S$ aureus were detected in their milk samples. mecA gene was detected in 2 coagulase positive and one coagulase negative $S$. aureus isolate making this study the first in Tanzania to report on coagulase negative $S$. aureus hourboring the mecA gene. Of the 3 MRSA isolates detected in the study only the coagulase negative $S$. aureus isolate was able to be characterized as spa type $t 2603$, whereas the other two MRSA's were spa un- typable.

S.aureus is one of the leading causes of bovine mastitis which explains the fact that the majority of publications included in this review concerning $S$. aureus in animals addressed the pathogen in clinical and sub-clinical mastitis or in regards to milk production quality. This has been instrumental in the reviews failure to make a link in describing animals and/or humans as potential $S$. aureus reservoir for each other, as well as the effect of such in both public and animal health. Very few studies on genomic $S$. aureus characterisation were available for review and did not suffice in showing such linkage.

\section{Limitations}

The data from this review cannot be generalized as true prevalence, antibiotic resistance patterns or genotypic characterization of $S$. aureus bacterium in Tanzania. High inter-study variations such as type of specimen analysed, objective of studies, time frame as well as methods employed including criteria set for resistance/MRSA confirmation may have influenced the outcome.

Methods used to genetically characterise $S$. aureus in this review were very divergent from simple PCR to whole genome sequencing. The data collected in these methods had different focuses which made comparing the results between studies difficult. Since the main objective in characterizing $S$. aureus or any other bacteria for that matter is understanding the genetic similarities and differences in conferring antibiotic resistance, strain type as well as and virulence factors, it would therefore be useful to have a common guideline that allowing inter laboratory comparability to gather more reliable and holistic data meant to guide treatment and infection prevention strategies in the country. 


\section{Conclusion}

Information about prevalence, antibiotic susceptibility and genotypic characteristics of $S$ aureus in Tanzania still remains scarce. It is evident that the majority of the publications included in this review are from research institutions or tertiary hospitals affiliated to universities with health related focuses hence most information could be derived from three focal points MNH in Dar es Salaam, BMC Mwanza and, KCMC Moshi respectively.

According to the Tanzania National Bureau of Statistics the focal regions represented in this review earn higher income per capital compared to the zones whereby no information could be gathered. This implies that most of the data obtained for review is based in relatively well to do regions (59). No published data between 2010 and 2018 on the epidemiology of S aureus was available for the southern, southern highlands, south western highlands as well as the western parts of the country, which is home to some of the most impoverished regions in Tanzania..

It is known that poverty struck areas also face other challenges as poor healthcare facilities and access, lack of basic needs such as food, proper housing and sanitation, which in turn leaves the population ridden by different infectious diseases. Furthermore since the income gained by the poor is mainly for subsistence, the tendency to resort to self-treatment and or consulting traditional healer is very high (60) all of which are known indicators associated with driving antibiotic resistance (61).

It is therefore imperative to emphasize the need to further gather information about the epidemiology (susceptibility patterns and genotypic characterization) of all bacteria of public health and animal health importance in Tanzania to gain a comprehensive description of the burden of the bacteria as well as enable proper strategies guiding empirical infections treatment and management in the country.

\section{Abbreviations}

AMR; Antimicrobial resistance, AST; Antibiotic susceptibility testing, BMC; Bugando Medical Centre, CAl: Community acquired Infection, CLSI; Clinical Laboratory Standard Institute, CNS;Coagulase negative Staphylococcus, HAl; Hospital Acquired Infection, HCW; Healthcare workers, ICU; Intensive care unit, KCMC; Kilimanjaro Christian Medical Centre; LAl; Livestock acquired infections, MLST; Multilocus sequence typing, MNH; Muhimbili National Hospital, MRSA; Methicillin resistant Staphylococcus aureus, MSSA; Methicillin sensitive Staphylococcus aureus, ND; Not done, NR; Not reported, NS; Not Screened, OM; Otitis media, RCH; Reproductive and child health, Spa; Staphylococcus protein A, SSI; Surgical Site infection, SSTI's; Skin and soft tissue infections, ST; Sequence type, UTI; Urinary tract infection, WHO: World Health Organization

\section{Declarations}

\section{Ethics approval and consent to participate}


Not applicable.

\section{Consent for publication}

Not applicable.

\section{Availability of data and materials}

Not applicable.

\section{Competing interests}

The authors declare that they have no competing interests.

\section{Funding}

This research was conducted under the PhD fellowship given by Consortium for Advanced Research Training in Africa (CARTA). CARTA is jointly led by the African Population and Health Research Center and the University of the Witwatersrand and funded by the Carnegie Corporation of New York (Grant numberB 8606.R02), Sida (Grant number 54100113), the DELTAS Africa Initiative (Grant number 107768/Z/15/Z) and Deutscher Akademischer Austauschdienst (DAAD). The DELTAS Africa Initiative is an independent funding scheme of the African Academy of Sciences (AAS)'s Alliance for Accelerating Excellence in Science in Africa (AESA) and supported by the New Partnership for Africa's Development Planning and Coordinating Agency (NEPAD Agency) with funding from the Wellcome Trust (UK) and the UK government. The statements made and views expressed are solely the responsibility of the Fellow.

\section{Authors' contributions}

TSM, TSK and JM participated in the literature search; TSM and TSK played equal part in the reviewing process of this work. TSM prepared the first draft of the manuscript. All authors read and approved the final manuscript

\section{Acknowledgements}

Not applicable.

\section{References}

1. O'Neill J. Tackling Drug-Resistant Infections Globally: Final Report and Recommendations. May 2016.

2. WHO. Antimicrobial resistance: Global report on surveillance Geneva; 2014. Contract No.: 9789241564748.

3. WHO. GLOBAL PRIORITY LIST OF ANTIBIOTIC-RESISTANT BACTERIA TO GUIDE RESEARCH, DISCOVERY, AND DEVELOPMENTOF NEW ANTIBIOTICS. 2017. 
4. Haddadin AS, Fappiano SA, Lipsett PA. Methicillin resistant Staphylococcus aureus (MRSA) in the intensive care unit. Postgrad Med J. 2002;78(921):385-92.

5. Jevons MP. “Celbenin” - resistant Staphylococci: Br Med J. 1961 Jan 14;1(5219):124-5.

6. Zetola N, Francis JS, Nuermberger EL, Bishai WR. Community-acquired meticillin-resistant Staphylococcus aureus: an emerging threat. Lancet Infect Dis. 2005;5(5):275-86.

7. Founou RC, Founou LL, Essack SY. Clinical and economic impact of antibiotic resistance in developing countries: A systematic review and meta-analysis. PloS one. 2017;12(12):e0189621.

8. David MZ DR. Community-Associated Methicillin-Resistant Staphylococcus aureus: Epidemiology and Clinical Consequences of an Emerging Epidemic. Clin Microbiol Rev. 2010;23::616-87.

9. Lozano C, Gharsa H, Ben Slama K, Zarazaga M, Torres C. Staphylococcus aureus in Animals and Food: Methicillin Resistance, Prevalence and Population Structure. A Review in the African Continent. Microorganisms. 2016;4(1):12.

10. Suleiman R. Local and regional variations in conditions for agriculture and food security in Tanzania: A review2018.

11. Joachim A, Moyo SJ, Nkinda L, Majigo M, Rugarabamu S, Mkashabani EG, et al. Nasal Carriage of Methicillin-Resistant Staphylococcus aureus among Health Care Workers in Tertiary and Regional Hospitals in Dar es Salam, Tanzania. International Journal of Microbiology. 2018;2018:7.

12. Geofrey A, Abade A, Aboud S. Methicillin-resistant Staphylococcus aureus (MRSA) colonization among Intensive Care Unit (ICU) patients and health care workers at Muhimbili national hospital, Dar Es Salaam, Tanzania, 2012. The Pan African Medical Journal. 2015;21.

13. Joachim A, Moyo SJ, Nkinda L, Majigo M, Mmbaga E, Mbembati N, et al. Prevalence of methicillinresistant Staphylococcus aureus carriage on admission among patients attending regional hospitals in Dar es Salaam, Tanzania. BMC Res Notes. 2017;10(1):417.

14. Moyo SJ, Aboud S, Blomberg B, Mkopi N, Kasubi M, Manji K, et al. High nasal carriage of methicillinresistant Staphylococcus aureus among healthy Tanzanian under-5 children. Microbial drug resistance (Larchmont, NY). 2014;20.

15. Chochua S, D'Acremont V, Hanke C, Alfa D, Shak J, Kilowoko M, et al. Increased Nasopharyngeal Density and Concurrent Carriage of Streptococcus pneumoniae, Haemophilus influenzae, and Moraxella catarrhalis Are Associated with Pneumonia in Febrile Children. PloS one. 2016;11(12):e0167725.

16. Bloch EM, West SK, Mabula K, Weaver J, Mrango Z, Munoz B, et al. Antibiotic Resistance in Young Children in Kilosa District, Tanzania 4 Years after Mass Distribution of Azithromycin for Trachoma Control. The American Journal of Tropical Medicine and Hygiene. 2017;97(3):815-8.

17. Katakweba AS, Muhairwa AP, Espinosa-Gongora C, Guardabassi L, Mtambo MM, Olsen JE. spa typing and antimicrobial resistance of Staphylococcus aureus from healthy humans, pigs and dogs in Tanzania. Journal of infection in developing countries. 2016;10(2):143-8.

18. Moyo S, Aboud S, Kasubi M, Maselle SY. Bacteria isolated from bloodstream infections at a tertiary hospital in Dar es Salaam, Tanzania-antimicrobial resistance of isolates. South African medical 
journal = Suid-Afrikaanse tydskrif vir geneeskunde. 2010;100(12):835-8.

19. Makani J, Mgaya J, Balandya E, Msami K, Soka D, Cox SE, et al. Bacteraemia in sickle cell anaemia is associated with low haemoglobin: a report of 890 admissions to a tertiary hospital in Tanzania. $\mathrm{Br}$ J Haematol. 2015;171(2):273-6.

20. Mhada TV, Fredrick F, Matee MI, Massawe A. Neonatal sepsis at Muhimbili National Hospital, Dar es Salaam, Tanzania; aetiology, antimicrobial sensitivity pattern and clinical outcome. BMC Public Health. 2012;12:904.

21. Manyahi J, Matee MI, Majigo M, Moyo S, Mshana SE, Lyamuya EF. Predominance of multi-drug resistant bacterial pathogens causing surgical site infections in Muhimbili National Hospital, Tanzania. BMC Res Notes. 2014;7:500.

22. Kazimoto T, Abdulla S, Bategereza L, Juma O, Mhimbira F, Weisser M, et al. Causative agents and antimicrobial resistance patterns of human skin and soft tissue infections in Bagamoyo, Tanzania. Acta Trop. 2018;186:102-6.

23. Mwambete KD, Eulambius M. High Prevalence of Antibiotic-Resistant Otitis Media-Associated Bacterial Flora of Asymptomatic People Living with HIV at Morogoro Hospital, Tanzania. J Int Assoc Provid AIDS Care. 2018;17:2325958218759761.

24. Okamo B, Moremi N, Seni J, Mirambo MM, Kidenya BR, Mshana SE. Prevalence and antimicrobial susceptibility profiles of Staphylococcus aureus nasal carriage among pre-clinical and clinical medical students in a Tanzanian University. BMC Research Notes. 2016;9(1):1-6.

25. Kayange N, Kamugisha E, Mwizamholya DL, Jeremiah S, Mshana SE. Predictors of positive blood culture and deaths among neonates with suspected neonatal sepsis in a tertiary hospital, MwanzaTanzania. BMC pediatrics. 2010;10(1):1.

26. Ahmed M, Mirambo MM, Mushi MF, Hokororo A, Mshana SE. Bacteremia caused by multidrugresistant bacteria among hospitalized malnourished children in Mwanza, Tanzania: a cross sectional study. BMC Res Notes. 2017;10(1):62.

27. Mbunda F, McHembe MD, Chalya PL, Rambau P, Mshana SE, Kidenya BR, et al. Experiences with surgical treatment of chronic lower limb ulcers at a tertiary hospital in northwestern Tanzania: a prospective review of 300 cases. BMC Dermatol. 2012;12:17.

28. Moremi N, Mushi MF, Fidelis M, Chalya P, Mirambo M, Mshana SE. Predominance of multi-resistant gram-negative bacteria colonizing chronic lower limb ulcers (CLLUs) at Bugando Medical Center. BMC Research Notes. 2014;7(1):211.

29. Mawalla B, Mshana SE, Chalya PL, Imirzalioglu C, Mahalu W. Predictors of surgical site infections among patients undergoing major surgery at Bugando Medical Centre in Northwestern Tanzania. BMC Surg. 2011;11.

30. Mpogoro FJ, Mshana SE, Mirambo MM, Kidenya BR, Gumodoka B, Imirzalioglu C. Incidence and predictors of surgical site infections following caesarean sections at Bugando Medical Centre, Mwanza, Tanzania. Antimicrob Resist Infect Control. 2014;3:25. 
31. Chalya PL, Mabula JB, Dass RM, Ngayomela IH, Chandika AB, Mbelenge N, et al. Major limb amputations: a tertiary hospital experience in northwestern Tanzania. J Orthop Surg Res. 2012;7:18.

32. Gilyoma JM, Mabula JB, Chalya PL. Animal-related injuries in a resource-limited setting: experiences from a Tertiary health institution in northwestern Tanzania. World J Emerg Surg. 2013;8(1):7.

33. Marwa KJ, Mushi MF, Konje E, Alele PE, Kidola J, Mirambo MM. Resistance to Cotrimoxazole and Other Antimicrobials among Isolates from HIV/AIDS and Non-HIV/AIDS Patients at Bugando Medical Centre, Mwanza, Tanzania. AIDS Research and Treatment. 2015;2015:8.

34. Mushi MF, Mwalutende AE, Gilyoma JM, Chalya PL, Seni J, Mirambo MM, et al. Predictors of disease complications and treatment outcome among patients with chronic suppurative otitis media attending a tertiary hospital, Mwanza Tanzania. BMC Ear Nose Throat Disord. 2016;16:1.

35. Moremi N, Claus H, Mshana SE. Antimicrobial resistance pattern: a report of microbiological cultures at a tertiary hospital in Tanzania. BMC infectious diseases. 2016;16(1):756.

36. Ngocho J S AC, Sariko M, Mmbaga B T, Kibiki G S. . Bacterial Etiology of Respiratory Tract Infections among Ambulatory School Children in Moshi Municipality, Tanzania. Science Journal of Public Health. 2015;Vol. 3(No. 5):625-32.

37. Kinabo GD, van der Ven A, Msuya LJ, Shayo AM, Schimana W, Ndaro A, et al. Dynamics of nasopharyngeal bacterial colonisation in HIV-exposed young infants in Tanzania. Tropical medicine \& international health : TM \& IH. 2013;18(3):286-95.

38. Kassam NA, Damian DJ, Kajeguka D, Nyombi B, Kibiki GS. Spectrum and antibiogram of bacteria isolated from patients presenting with infected wounds in a Tertiary Hospital, northern Tanzania. BMC Research Notes. 2017;10(1):757.

39. Kumburu HH, Sonda T, Mmbaga BT, Alifrangis M, Lund O, Kibiki G, et al. Patterns of infections, aetiological agents and antimicrobial resistance at a tertiary care hospital in northern Tanzania. Tropical medicine \& international health : TM \& IH. 2017;22(4):454-64.

40. Mahende C, Ngasala B, Lusingu J, Butichi A, Lushino P, Lemnge M, et al. Aetiology of acute febrile episodes in children attending Korogwe District Hospital in north-eastern Tanzania. PloS one. 2014;9(8):e104197.

41. Caggiano S, Ullmann N, De Vitis E, Trivelli M, Mariani C, Podagrosi M, et al. Factors That Negatively Affect the Prognosis of Pediatric Community-Acquired Pneumonia in District Hospital in Tanzania. Int J Mol Sci. 2017;18(3).

42. Onken A, Said AK, Jorstad M, Jenum PA, Blomberg B. Prevalence and Antimicrobial Resistance of Microbes Causing Bloodstream Infections in Unguja, Zanzibar. PloS one. 2015;10(12):e0145632.

43. Thriemer K, Ley B, Ame S, von Seidlein L, Pak GD, Chang NY, et al. The burden of invasive bacterial infections in Pemba, Zanzibar. PloS one. 2012;7(2):e30350.

44. Hudzicki J. Disk Diffusion Susceptibility Test Protocol. Kirby-Bauer. Amecan Society for Microbiology. 2009.

45. Reller LB, Weinstein M, Jorgensen JH, Ferraro MJ. Antimicrobial Susceptibility Testing: A Review of General Principles and Contemporary Practices. Clinical Infectious Diseases. 2009;49(11):1749-55. 
46. den Heijer CD, van Bijnen EM, Paget WJ, Pringle M, Goossens H, Bruggeman CA, et al. Prevalence and resistance of commensal Staphylococcus aureus, including meticillin-resistant $S$ aureus, in nine European countries: a cross-sectional study. The Lancet Infectious Diseases. 2013;13(5):409-15.

47. Lestari ES, Severin, J.A., Filius, P.M.G. FiliusK. KuntamanD. O. DuerinkU. HadiH. WahjonoH. A. Verbrugh Antimicrobial resistance among commensal isolates of Escherichia coli and Staphylococcus aureus in the Indonesian population inside and outside hospitals. Eur J Clin Microbiol Infect Dis. (2008);27(45).

48. Shittu AO, Lin J. Antimicrobial susceptibility patterns and characterization of clinical isolates of Staphylococcus aureus in KwaZulu-Natal province, South Africa. BMC infectious diseases. 2006;6:125.

49. Nwankwo Emmanuel Onwubiko NMS. Antibiotic sensitivity pattern of Staphylococcus aureus from clinical isolates in a tertiary health institution in Kano, Northwestern Nigeria. The Pan African Medical Journal. 2011;8(4).

50. Abdulgader SM, Shittu AO, Nicol MP, Kaba M. Molecular epidemiology of Methicillin-resistant Staphylococcus aureus in Africa: a systematic review. Frontiers in microbiology. 2015;6:348.

51. Moremi N, Mshana SE, Kamugisha E, Kataraihya J, Tappe D, Vogel U, et al. Predominance of methicillin resistant Staphylococcus aureus-ST88 and new ST1797 causing wound infection and abscesses. Journal of infection in developing countries. 2012;6(8):620-5.

52. Nurjadi D, Olalekan AO, Layer F, Shittu AO, Alabi A, Ghebremedhin B, et al. Emergence of trimethoprim resistance gene dfrG in Staphylococcus aureus causing human infection and colonization in subSaharan Africa and its import to Europe. Journal of Antimicrobial Chemotherapy. 2014;69(9):2361-8.

53. Ruffing U, Alabi A, Kazimoto T, Vubil DC, Akulenko R, Abdulla S, et al. Community-Associated Staphylococcus aureus from Sub-Saharan Africa and Germany: A Cross-Sectional Geographic Correlation Study. Scientific reports. 2017;7(1):154.

54. Mohammed J ZMH, Hounmanou Y M „, Kisanga A aTHN. Molecular Typing and Antimicrobial Susceptibility of Methicillin-Resistant Staphylococcus aureus Isolated from Bovine Milk in Tanzania. Hindawi International Journal of Microbiology. 2018;2018,.

55. Kashoma I.P. LEP MJ, Mtemela B.O and Medardus J.J. Prevalence and antimicrobial susceptibility profiles of Staphylococcus aureus from cow's milk, nasal and environmental swabs in selected dairy farms in Morogoro, Tanzania. Tanzania Veterinary Journal. 2018;30(2):61 -75.

56. Suleiman TS KE, Mdegela RH. . Prevalence of bovine subclinical mastitis and antibiotic susceptibility patterns of major mastitis pathogens isolated in Unguja island of Zanzibar, Tanzania. Trop Anim Health Prod. 2018;50(2):259-66.

57. Ngasala JH NH, Madundo M, Mtambo A. Assessment of raw milk quality and stakeholders'awareness on milk-borne health risks in Arusha City and Meru District, Tanzania. Tropical Animal Health and Production. 2015.

58. Caudell MA QM, Subbiah M, Call, DR RC, Roulette JW, et al. . Antimicrobial Use and Veterinary Care among Agro-Pastoralists in Northern Tanzania. PloS one. (2017);12(1). 
59. HIGHLIGHTS FOR THE THIRD QUARTER (JULY - SEPTEMBER) GROSS DOMESTIC PRODUCT, 2017. United republic of Tanzania: National Bureau of Statistics Ministry of Finance and Planning; 2018.

60. Green JA, Norris P, Bukhari NI. Self-medication, home remedies, and spiritual healing: common responses to everyday symptoms in Pakistan AU - Anwar, Mudassir. Health Psychology and Behavioral Medicine. 2015;3(1):281-95.

61. Byarugaba DK. A view on antimicrobial resistance in developing countries and responsible risk factors. International journal of antimicrobial agents. 2004;24(2):105-10.

\section{Figures}

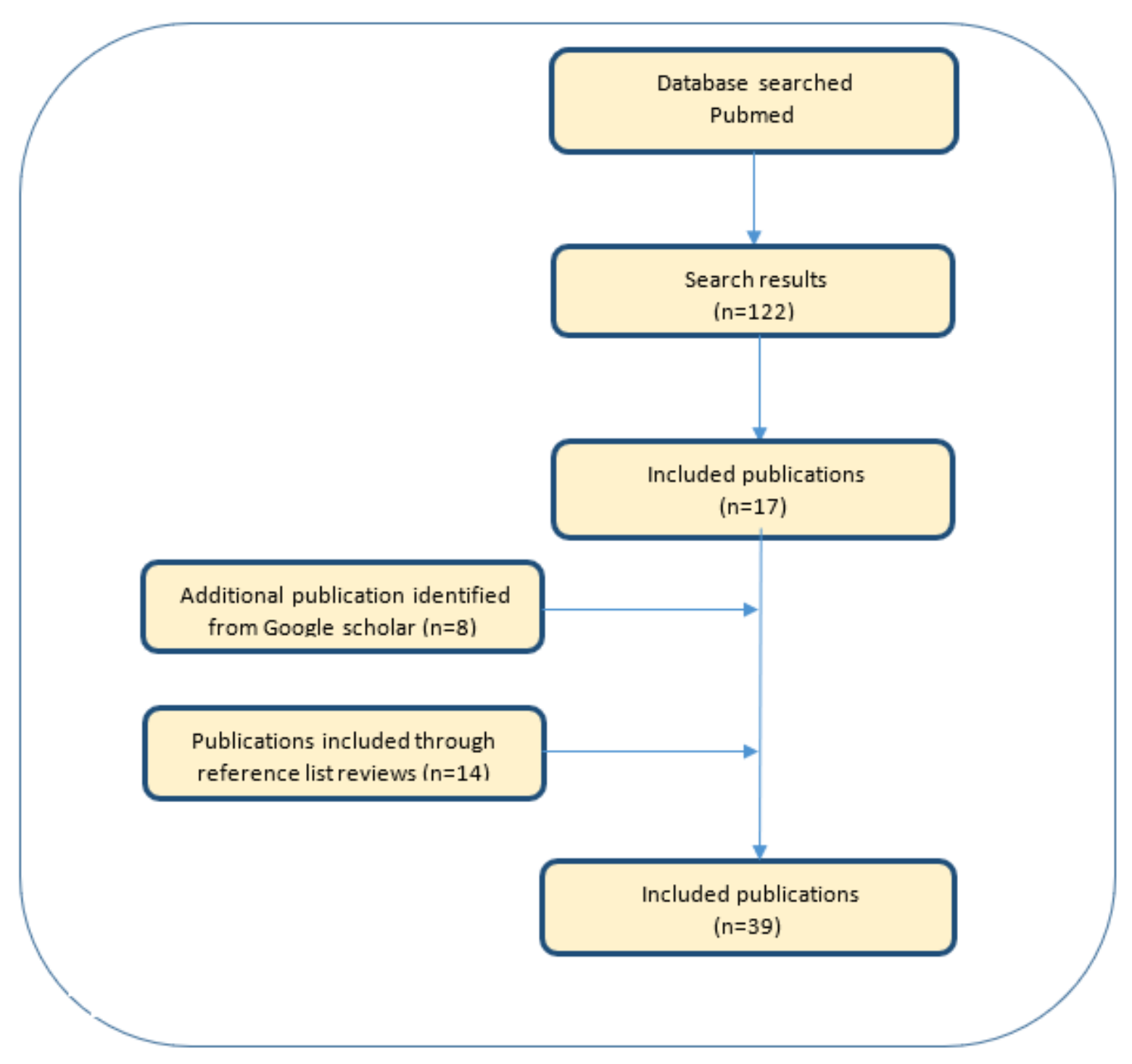

Figure 1

PRISMA diagram for a systematic review examining prevalence, antimicrobial resistance and genotyping characteristics of $\mathrm{S}$. aureus in Tanzania 


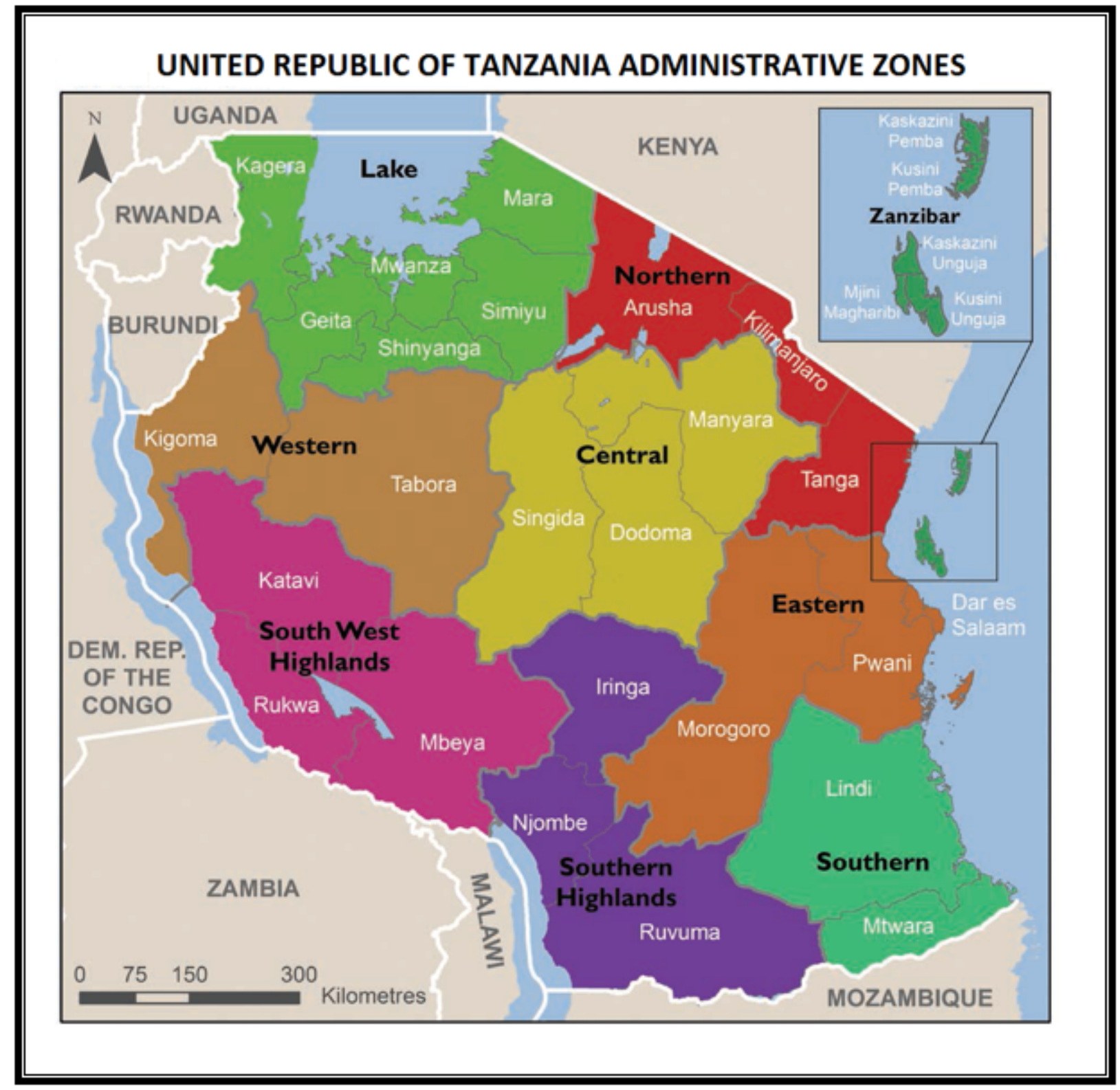

Figure 2

Tanzania administrative zones. Modified map adopted from Suleiman 2018 (10) 


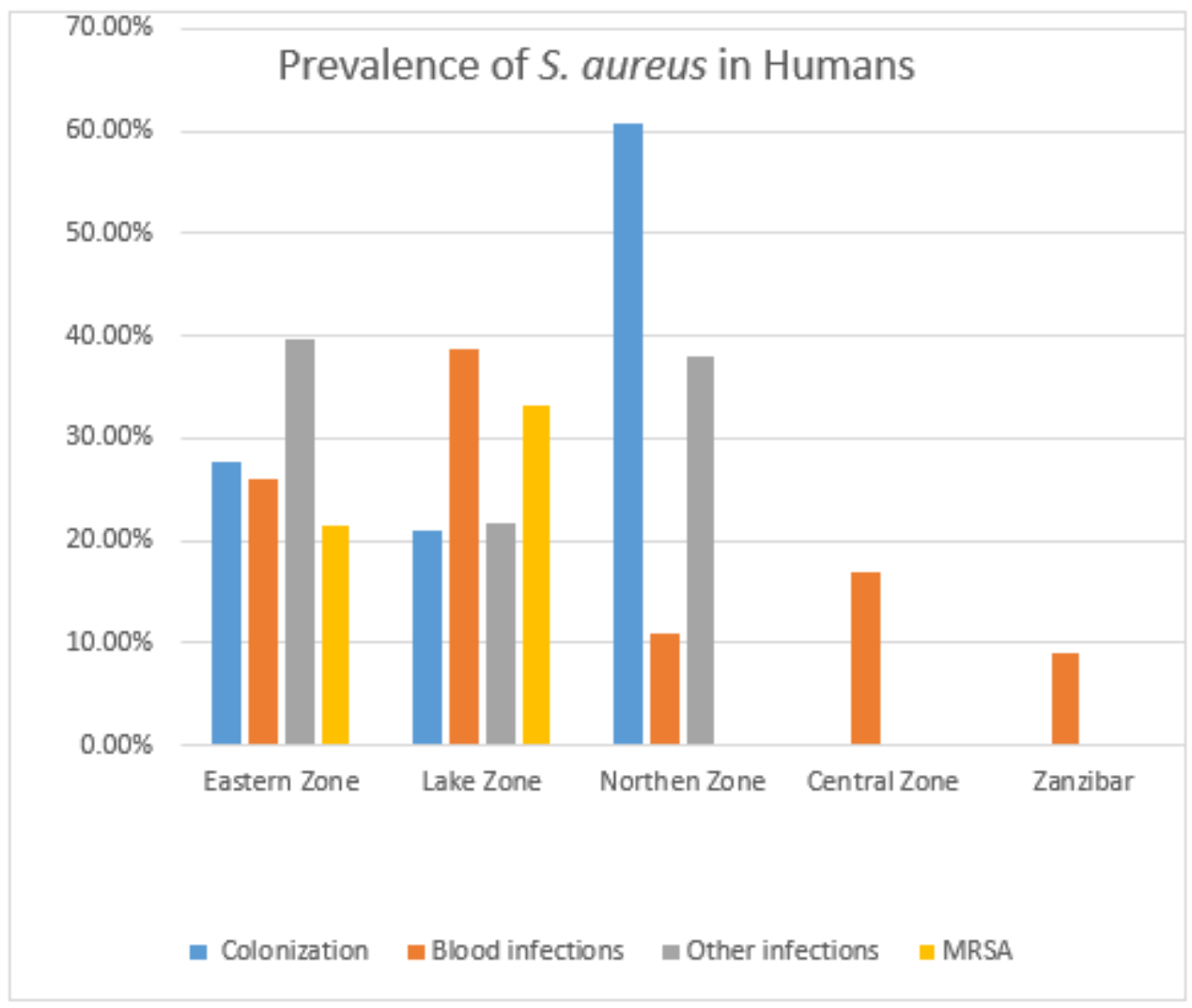

\section{Figure 3}

Distribution of S. aureus prevalence in colonization and different infections from humans isolates based on reviewed publications in Tanzania. Regional variations in prevalence of different infections could be observed. S. aureus blood was more prevalent in the lake zone compared to other regions. Collectively the review revealed that $\mathrm{S}$. aureus was most frequently implicated in association with skin and soft tissue infections. Prevalence in colonized people in all reported regions was generally normal with exception to the northern zone where the prevalence was uncharacteristically high.

\section{Supplementary Files}

This is a list of supplementary files associated with this preprint. Click to download.

- PRISMA2009checklistReview.doc 